Draft Version September 4, 2018

Preprint typeset using $\mathrm{LAT}_{\mathrm{E} X}$ style emulateapj v. 6/22/04

\title{
MAGNETIC BURIAL AND THE HARMONIC CONTENT OF MILLISECOND OSCILLATIONS IN THERMONUCLEAR X-RAY BURSTS
}

\author{
D. J. B. Payne and A. Melatos \\ School of Physics, University of Melbourne, Parkville, VIC 3010, Australia. \\ Draft version September 4, 2018
}

\begin{abstract}
Matter accreting onto the magnetic poles of a neutron star spreads under gravity towards the magnetic equator, burying the polar magnetic field and compressing it into a narrow equatorial belt. Steady-state, Grad-Shafranov calculations with a self-consistent mass-flux distribution (and a semiquantitative treatment of Ohmic diffusion) show that, for $M_{\mathrm{a}} \gtrsim 10^{-5} M_{\odot}$, the maximum field strength and latitudinal half-width of the equatorial magnetic belt are $B_{\max }=5.6 \times 10^{15}\left(M_{\mathrm{a}} / 10^{-4} M_{\odot}\right)^{0.32} \mathrm{G}$ and $\Delta \theta=\max \left[3^{\circ}\left(M_{\mathrm{a}} / 10^{-4} M_{\odot}\right)^{-1.5}, 3^{\circ}\left(M_{\mathrm{a}} / 10^{-4} M_{\odot}\right)^{0.5}\left(\dot{M}_{\mathrm{a}} / 10^{-8} M_{\odot} \mathrm{yr}^{-1}\right)^{-0.5}\right]$ respectively, where $M_{\mathrm{a}}$ is the total accreted mass and $\dot{M}_{\mathrm{a}}$ is the accretion rate. It is shown that the belt prevents north-south heat transport by conduction, convection, radiation, and ageostrophic shear. This may explain why millisecond oscillations observed in the tails of thermonuclear (type I) X-ray bursts in low-mass X-ray binaries are highly sinusoidal: the thermonuclear flame is sequestered in the magnetic hemisphere which ignites first. The model is also consistent with the occasional occurrence of closely spaced pairs of bursts. Time-dependent, ideal-magnetohydrodynamic simulations confirm that the equatorial belt is not disrupted by Parker and interchange instabilities.
\end{abstract}

Subject headings: accretion, accretion disks — stars: magnetic fields — stars: neutron — stars: rotation - X-rays: bursts

\section{INTRODUCTION}

Thermonuclear (type I) X-ray bursts are observed from 70 of the 160 low-mass X-ray binaries (LMXBs) discovered to date (Strohmaver \& Bildsten 2003). They recur every few hours to days, when the accreted surface layer of the neutron star ignites and is incinerated by hydrogen and helium burning. Brightness oscillations with millisecond periods are observed during thermonuclear X-ray bursts in 13 LMXBs (Muno et al. 2002; Piro \& Bildsten 2004). They arise during burst onset because the stellar photosphere is temporarily patchy while the thermonuclear flame spreads from its ignition point to cover the star.

A surprising property of the burst oscillations is that they often persist into the tails of bursts, after the flame is expected to have engulfed the star (Strohmaver et al. 1997). Equally surprising is how sinusoidal the oscillations are. Muno et al. (2002) analyzed the harmonic content of 59 oscillations from six sources, observed with the Rossi X-Ray Timing Explorer (RXTE), and found that the Fourier amplitudes of integer and half-integer harmonics are less than 5 and 10 per cent of the maximum signal respectively. These data imply that if there is one hot spot on the surface, it must lie near the rotational pole or cover an entire hemisphere, whereas if there are two, antipodal hot spots, they must lie near the rotational equator (Muno et al. 2002). Most theories that explain why the oscillations persist into the tails of the bursts, e.g. uneven heating/cooling during photospheric uplift (Strohmaver et al. 1997), or cyclones driven by zonal shear in a geostrophic flow (Spitkovsky et al. 2002), are hard pressed to account for the pattern of hot spots implied by the Fourier data. Global $r$-modes in the neutron star ocean can divide the photosphere into symmet-

Electronic address: dpayne@physics.unimelb.edu.au ric halves (Hevl 2004), but the physical mechanism converting $r$-mode density perturbations to brightness oscillations is unclear (Muno et al. 2002). Cumming (2005) showed that differential rotation between the pole and equator of $\gtrsim 2$ per cent can also excite unstable modes.

In this paper, we show that an equatorial belt of intense magnetic field, compressed by accreted material spreading away from the magnetic poles, can impede thermal transport between the hemispheres of the star. In $\S 2$, we review the physics of magnetic burial and compute the maximum field strength and width of the equatorial magnetic barrier. In $\S 3$, we estimate the efficiency of thermal transport across the barrier by conduction, convection, radiation, and ageostrophic shear, and investigate whether cyclonic flows can disrupt the barrier. The implications for the harmonic content of burst oscillations are explored in $\S 4$.

\section{MAGNETIC BURIAL}

\subsection{Grad-Shafranov equilibria}

In the process of magnetic burial, material accreting onto a neutron star accumulates in a column at the magnetic polar cap, until the hydrostatic pressure at the base of the column overcomes the magnetic tension and matter spreads equatorward, dragging along frozen-in polar magnetic field lines to form an equatorial magnetic belt or 'tutu'. Figure 1(a), reproduced from Pavne \& Melatos (2004), illustrates the equilibrium configuration obtained for $M_{\mathrm{a}}=10^{-5} M_{\odot}$, where $M_{\mathrm{a}}$ is the total accreted mass. The polar mountain of accreted material (dashed contours) and the pinched, flaring, equatorial magnetic belt are evident (Melatos \& Phinnev 2001; Pavne \& Melatos 2004). The equatorial magnetic field strength increases in inverse proportion to the surface area of the equatorial belt, by flux conservation. The Lorentz force per unit volume exerted by the compressed equatorial field [Fig- 
ure 1(b)] balances the thermal pressure gradient [Figure [1(c)], and gravity, preventing the accreted matter from spreading to the equator.

In the steady state, the equations of ideal magnetohydrodynamics (MHD) reduce to the force balance equation (CGS units)

$$
\nabla p+\rho \nabla \phi-(4 \pi)^{-1}(\nabla \times \mathbf{B}) \times \mathbf{B}=0,
$$

where $\mathbf{B}, \rho, p=c_{\mathrm{s}}^{2} \rho$, and $\phi(r)=G M_{*} r / R_{*}^{2}$ denote the magnetic field, fluid density, pressure, and gravitational potential respectively, $c_{\mathrm{S}}$ is the isothermal sound speed, $M_{*}$ is the mass of the star, and $R_{*}$ is the stellar radius. In spherical polar coordinates $(r, \theta, \phi)$, for an axisymmetric field $\mathbf{B}=\nabla \psi(r, \theta) /(r \sin \theta) \times \hat{\mathbf{e}}_{\phi}$, equation (1) reduces to the Grad-Shafranov equation

$$
\Delta^{2} \psi=F^{\prime}(\psi) \exp \left[-\left(\phi-\phi_{0}\right) / c_{\mathrm{s}}^{2}\right],
$$

where

$$
\Delta^{2}=\frac{1}{\mu_{0} r^{2} \sin ^{2} \theta}\left[\frac{\partial^{2}}{\partial r^{2}}+\frac{\sin \theta}{r^{2}} \frac{\partial}{\partial \theta}\left(\frac{1}{\sin \theta} \frac{\partial}{\partial \theta}\right)\right]
$$

is the Grad-Shafranov operator, $F(\psi)$ is an arbitrary function of the magnetic flux $\psi$, and we set $\phi_{0}=\phi\left(R_{*}\right)$. In this paper, as in Pavne \& Melatos (2004), we fix $F(\psi)$ uniquely by connecting the initial dipolar state (with $M_{\mathrm{a}}=0$ ) and final distorted state [e.g. Figure 1(a)] via the integral form of the flux freezing condition of ideal MHD, viz.

$$
\frac{d M}{d \psi}=2 \pi \int_{C} \frac{d s \rho}{|\mathbf{B}|} .
$$

Here, $C$ is any magnetic field line, and the mass-flux distribution $d M / d \psi$ is chosen to capture the magnetospheric geometry, e.g. the accretion stream is funneled magnetically onto the pole, with $d M / d \psi \propto \exp \left(-\psi / \psi_{\mathrm{a}}\right)$, where $\psi_{\mathrm{a}}$ is the polar flux. We also assume north-south symmetry and adopt the boundary conditions $\psi=$ dipole at $r=R_{*}$ (line tying), $\psi=0$ at $\theta=0$, and $\partial \psi / \partial r=0$ at large $r$. The line-tying approximation artificially prevents accreted material from sinking, so the computed $\rho$ is an upper limit. Equations (2) and (3) are solved numerically using an iterative relaxation scheme and analytically by Green functions, producing equilibria like Figure 1.

\subsection{Equatorial magnetic belt}

The compressed magnetic field in the equatorial belt emerges approximately perpendicular to the stellar surface, with opposite sign in the two hemispheres. Near the equator, $B$ falls off roughly as $\exp [-0.7(\pi / 2-$ $\theta) / \Delta \theta] \exp \left[-\left(r-R_{*}\right) / h\right]$, where $h=c_{\mathrm{s}}^{2} R_{*} / G M_{*}$ is the hydrostatic scale height, $\Delta \theta$ is the belt thickness, and the factor 0.7 comes from empirically fitting to the numerical results. The maximum magnetic field strength in the belt, $B_{\max }$, computed numerically as a function of $M_{\mathrm{a}}$, is plotted in Figure 2 It is fitted by

$B_{\max }= \begin{cases}2.0 \times 10^{16}\left(M_{\mathrm{a}} / M_{\mathrm{c}}\right)^{0.91 \pm 0.06} \mathrm{G} & M_{\mathrm{a}} \lesssim 0.4 M_{\mathrm{c}} \\ 6.3 \times 10^{15}\left(M_{\mathrm{a}} / M_{\mathrm{c}}\right)^{0.32 \pm 0.01} \mathrm{G} & M_{\mathrm{a}} \gtrsim 0.4 M_{\mathrm{c}}\end{cases}$

with $M_{\mathrm{c}}=G M_{*} B_{0}^{2} R_{*}^{2} / 8 c_{\mathrm{s}}^{4} \sim 10^{-4} M_{\odot}$, where $B_{0}$ is the polar magnetic field strength prior to accretion. The scaling (5.) agrees with analytic theory for small $M_{\mathrm{a}}$
(Pavne \& Melatos 2004). Also plotted in Figure 2 is the half-width half-maximum thickness of the belt, fitted by $\Delta \theta=3^{\circ}\left(M_{\mathrm{a}} / M_{\mathrm{c}}\right)^{-1.5 \pm 0.03}$. We find that $\Delta \theta$ decreases as $B_{\max }$ increases, as expected from magnetic flux conservation, but not exactly as $\Delta \theta \propto B_{\max }^{-1}$, because $B_{r}$ is underestimated numerically at the equator by $\sim 10$ per cent (flux loss due to finite grid resolution). Note that equation (5) and the above scalings of $\Delta \theta$ versus $M_{\mathrm{a}}$ do not include Ohmic diffusion, which is discussed further in $\S 2.3$ and $\$ 4$

Grad-Shafranov equilibria are difficult to compute directly from (2) and (4) for $M_{\mathrm{a}} \gtrsim 1.6 M_{\mathrm{c}}$, because the magnetic topology changes abruptly: magnetic bubbles form which are disconnected from the surface, hinting at time-dependent processes which the steady-state theory cannot describe. In addition, the iterative relaxation scheme struggles to handle steep field gradients. Therefore the results in Figure 2 for $M_{\mathrm{a}} \geq 1.6 M_{\mathrm{c}}$ are computed by direct numerical simulation using ZEUS$3 \mathrm{D}$, a multipurpose, time-dependent, ideal-MHD code for astrophysical fluid dynamics which uses staggeredmesh finite differencing and operator splitting in three dimensions (Stone \& Norman 1992). We load the GradShafranov equilibrium for $M_{\mathrm{a}}=1.6 M_{\mathrm{c}}$ into ZEUS-3D; double $M_{\mathrm{a}}$ quasistatically over 250 Alfvén times with inflow (and hence radial $\mathbf{B}$ ) boundary conditions; stop the inflow and allow $\mathbf{B}$ to relax to a dipole at large $r$; then iterate to reach $M_{\mathrm{a}} \sim 10^{-3} M_{\odot}$ (Pavne \& Melatos 2006). An isothermal equation of state is chosen and self-gravity is switched off. The experiment is performed for $h / R_{*}=2 \times 10^{-2}$ (for computational efficiency) and scaled to neutron star conditions $\left(h / R_{*}=5 \times 10^{-5}\right)$ according to $B_{\max } \propto R_{*} / h$; this scaling is verified numerically for the range of $M_{\mathrm{a}}$ in Figure 2

\subsection{Stability and Ohmic relaxation}

Distorted ideal-MHD equilibria are often disrupted by Parker and interchange instabilities. Remarkably, however, this is not true for the equilibrium in Figure 1 Figure 3 shows the results of an experiment in which the equilibrium is loaded into ZEUS-3D, perturbed, and evolved for 500 Alfvén times (Pavne \& Melatos 2006). [The Alfvén time is defined as $h / v_{\mathrm{A}}$, where $v_{\mathrm{A}}$ is the Alfvén speed averaged over the grid.] It is marginally stable; $B_{\max }$ oscillates via magnetosonic (phase speed $\approx c_{\mathrm{s}}$ ) and Alfvén (phase speed $\approx v_{\mathrm{A}}$ ) modes which are damped by numerical dissipation. The configuration already represents the end point (reached quasistatically) of the nonlinear Parker instability of an initially dipolar field overlaid with accreted material. It is not interchange unstable because line tying prevents flux tubes from squeezing past each other. That said, we caution that we have not yet investigated the full gamut of three-dimensional MHD instabilities; cross-field mass transport cannot be categorically ruled out.

Another pathway to cross-field mass transport is if the magnetic belt relaxes by Ohmic diffusion, either during quiescence or during a burst itself. During quiescence, diffusion occurs most rapidly at the base of the accreted layer. In the relaxation time approximation, with Coulomb logarithms set to 10, the electron-phonon conductivity for a hydrogen-helium mixture is $\sigma=6.3 \times$ $10^{24}\left(\rho / 10^{11} \mathrm{~g} \mathrm{~cm}^{-3}\right) \mathrm{s}^{-1}$, the density at the base of the accreted layer is $\rho=6.2 \times 10^{10}\left(M_{\mathrm{a}} / 10^{-5} M_{\odot}\right)^{3 / 4} \mathrm{~g} \mathrm{~cm}^{-3}$ 
(Brown \& Bildsten 1998), and hence the Ohmic diffusion time-scale across the equatorial belt (at the base of the accreted layer) is $t_{\mathrm{d}}=4 \pi \sigma R_{*}^{2} \Delta \theta^{2} / c^{2}=2.6 \times$ $10^{7}\left(M_{\mathrm{a}} / 10^{-5} M_{\odot}\right)^{-9 / 4}\left(T / 10^{8} \mathrm{~K}\right)^{-1} \mathrm{yr}$, much longer than the burst time-scale, where we rewrite $\Delta \theta$ in terms of $M_{\mathrm{a}}$. During a burst, diffusion occurs most rapidly in the burning layer. The temperature of the burning layer rises isobarically until the radiation pressure dominates the hydrostatic pressure, reaching $T \sim 10^{8} \mathrm{~K}$ for a typical ignition column (Brown 2004). The elevated temperature and reduced density lower $\sigma$ and accelerate Ohmic diffusion. In the burning layer $\left(\rho \approx 10^{6} \mathrm{~g} \mathrm{~cm}^{-3}\right)$, we find $t_{\mathrm{d}}=1.5 \times 10^{3} \mathrm{yr}$, still much longer than the burst time-scale.

Ohmic diffusion will be modelled self-consistently in a future paper.

\section{THERMAL TRANSPORT ACROSS THE EQUATOR}

Does the equatorial magnetic belt impede thermal transport enough to stop the thermonuclear flame in a type I X-ray burst from spreading from one hemisphere to another? A burst is initiated locally by a thin shell thermal instability (Schwarzschild \& Härm 1965). As the nuclear burning time-scale is much shorter than the time to accrete the minimum column for ignition, the accreted layer ignites at a single point, most likely at the equator where gravity is rotationally reduced, and the thermonuclear flame spreads away either as a deflagration front (Frvxell \& Wooslev 1982; Bildsten 1995), by detonation (Frvxell \& Wooslev 1982; Zingale et al. 2001), or as a cyclone driven by zonal shear (Spitkovskv et al. 2002). Detonation, which occurs when the nuclear burning time-scale is less than the vertical sound crossing time-scale, requires a thick $(\sim 100 \mathrm{~m})$ column of fuel and hence a low accretion rate $\left(\lesssim 10^{-11.5} M_{\odot}\right.$ $\left.\mathrm{yr}^{-1}\right)$. Deflagration occurs most commonly, with the front propagating at a speed set by the heat flux and convection (Frvxell \& Wooslev 1982). Note that temperature fluctuations leading to single-point ignition are difficult to create because the sound crossing time around the star is very short, but Coriolis forces can assist by balancing sideways pressure gradients when the star is rapidly rotating (Spitkovskv et al. 2002).

We estimate below how the equatorial magnetic belt modifies heat transport in these scenarios.

\subsection{Conduction}

The thermal conductivity $\kappa$ of degenerate electrons at the atmosphere-ocean boundary of a magnetized neutron star, where $\mathrm{H} / \mathrm{He}$ burning occurs, was calculated by Potekhin (1999). We extract approximate values for the conductivity perpendicular to the magnetic field from Figures 5 and 6 of that paper, obtaining $\kappa_{\perp} \approx$ $10^{7.5}\left(\rho / 10^{6} \mathrm{~g} \mathrm{~cm}^{-3}\right)\left(T / 10^{8} \mathrm{~K}\right)^{2}\left(B / 10^{15} \mathrm{G}\right)^{-2}$ in units of erg s$~^{-1} \mathrm{~cm}^{-1} \mathrm{~K}^{-1}$ in the regime $10^{4} \mathrm{~g} \mathrm{~cm}^{-3} \lesssim \rho \lesssim 10^{9}$ $\mathrm{g} \mathrm{cm}^{-3}, 10^{12} \mathrm{G} \lesssim B \lesssim 10^{15} \mathrm{G}$, and $10^{6} \mathrm{~K} \underset{\lesssim}{\approx} \lesssim 10^{8}$ $\mathrm{K}$. Note that $\kappa_{\perp} \approx$ at the equator is reduced $10^{6}$ times relative to its value before accretion commences $\left(\propto B^{-2}\right)$ and, for reference, one has $\kappa_{\perp} / \kappa_{\|} \sim 10^{-5}$ at $T=10^{8} \mathrm{~K}$, $B=10^{12} \mathrm{G}$ (Potekhin 1999). We estimate the conduction time-scale across the magnetic barrier from $t_{\text {cond }}=$ $R_{*}^{2} \Delta \theta^{2} C \rho / \kappa_{\perp}$, where $C=10^{4}\left(T / 10^{8} \mathrm{~K}\right) \mathrm{erg} \mathrm{g}^{-1} \mathrm{~K}^{-1}$ is the specific heat capacity of a degenerate Fermi gas
(Brown \& Bildsten 1998), finding

$$
t_{\text {cond }}=27\left(M_{\mathrm{a}} / M_{\mathrm{c}}\right)^{-3}\left(T / 10^{8} \mathrm{~K}\right)^{-1}\left(B / 10^{15} \mathrm{G}\right)^{2} \mathrm{yr},
$$

which is safely longer than the duration of the burst. Note that this estimate does not include corrections due to electron-electron scattering and impurity scattering. Furthermore, our fit smoothes over the wiggles in the solid curves of Figures 5 and 6 in Potekhin (1999), which arise from quantization into Landau orbitals.

\subsection{Radiation}

In a strong magnetic field, photons polarized perpendicular to $\mathbf{B}$ dominate radiative transport. In the burning (helium) layer, where Thomson scattering dominates, the Rosseland mean opacity $\perp \mathbf{B}$ is given by $\kappa_{\mathrm{R}}=$ $1.3 \times 10^{-6}\left(T / 10^{8} \mathrm{~K}\right)^{2}\left(B / 10^{15} \mathrm{G}\right)^{-2} \mathrm{~g}^{-1} \mathrm{~cm}^{2}$, decreasing in inverse proportion to the square of the cyclotron frequency $\left(\omega_{c}\right)$ (Joss \& Li 1980; Frvxell \& Wooslev 1982). Hence the optical depth of the barrier is $\tau_{\mathrm{R}}=\kappa_{\mathrm{R}} \rho R_{*} \Delta \theta$, i.e.

$$
\tau_{\mathrm{R}}=6.8 \times 10^{4}\left(M_{\mathrm{a}} / M_{\mathrm{c}}\right)^{-3 / 2}\left(T / 10^{8} \mathrm{~K}\right)^{2}\left(B / 10^{15} \mathrm{G}\right)^{-2} .
$$

The optical depth (77) is a lower limit obtained by assuming that radiation is transported from one hemisphere to the other near the surface, at the depth of the burning layer $\left(\rho \sim 10^{6} \mathrm{~g} \mathrm{~cm}^{-3}\right)$ rather than at the base of the accreted column $\left(\rho \sim 10^{11} \mathrm{~g} \mathrm{~cm}^{-3}\right)$. Hence the peak flux penetrating the barrier is $F_{\text {burst }}=L_{\text {burst }} e^{-\tau_{\mathrm{R}}} / 4 \pi R_{*}^{2}=$ $8 \times 10^{24} e^{-\tau_{\mathrm{R}}} \mathrm{erg} \mathrm{s}^{-1} \mathrm{~cm}^{-2}$ (for $L_{\text {burst }}=10^{38} \mathrm{erg} \mathrm{s}^{-1}$ ), which is insufficient to heat the other (quiescent) hemisphere above its ignition temperature $\left(\sim 10^{8} \mathrm{~K}\right)$ (assuming thermal equilibrium and applying the StefanBoltzmann law). Interestingly, once $B_{\max }$ exceeds $10^{17}$ G, we find $\tau_{\mathrm{R}} \lesssim 1$ and the magnetic belt becomes optically thin. Vertical heat propagation is not considered here.

Equation (77) can be generalized in several ways. Mode coupling (Miller 1995) can cause a fraction $\sim 0.2 \omega / \omega_{\mathrm{c}}$ of the perpendicular mode photons to convert into parallel mode photons, so that the opacity scales $\propto B^{-1}$, increasing the field strength required for the belt to become optically thin. Vacuum polarization (Ozel 2003), along with proton-cyclotron resonance, gives sharp spikes in the frequency response of the atmospheric opacity. However, the resonant densities for vacuum polarization $\left(\rho \sim 10^{-3}\right.$ $\mathrm{g} \mathrm{cm}^{-3}$ ) are achieved at shallow depths, well above the burning layer.

\subsection{Convection}

Bildsten (1995) estimated the convective speed in the burning layer in terms of the mixing length, $l_{\mathrm{m}}$, and thermal time-scale, $t_{\mathrm{th}}$, finding $v_{\mathrm{c}} \approx$ $c_{\mathrm{s}}\left(l_{\mathrm{m}} / h\right)^{1 / 3}\left(c_{\mathrm{s}} R_{*}^{2} / G M_{*} t_{\mathrm{th}}\right)^{1 / 3} \approx 10^{6} \mathrm{~cm} \mathrm{~s}^{-1}$. This is consistent with the upper bound $10^{7} \mathrm{~cm} \mathrm{~s}^{-1}$ obtained if $L_{\text {burst }}$ is transported entirely by the mechanical flux $\rho v_{\mathrm{c}}^{3}$. It is also consistent with the observed burst rise time $R_{*} / v_{\mathrm{c}} \lesssim 1 \mathrm{~s}$ (Spitkovskv et al. 2002). Convection is stabilized magnetically if the magnetic tension exceeds the ram pressure $\rho v_{\mathrm{c}}^{2}$, which occurs for $B_{\max } \gtrsim\left(8 \pi \rho v_{\mathrm{c}}^{2}\right)^{1 / 2} \approx$ $5 \times 10^{9} \mathrm{G}$. This condition is met comfortably in the equatorial belt. The magnetic field can also quench convection (Gough \& Tavler 1966). 


\subsection{Ageostrophic shear flow}

Spitkovskv et al. (2002) suggested that inhomogeneous cooling drives zonal currents which are unstable to the formation of cyclones, as in planetary atmospheres. This may explain why the coherent oscillations observed in the tails of some type I X-ray bursts persist for many rise times. The drift in oscillation frequency $(\sim \mathrm{Hz})$ during the burst is attributed to the Coriolis drift of the cyclone in the frame of the star, although theory predicts larger frequency drifts $(\sim 10 \mathrm{~Hz})$ than those observed. The oscillation amplitude in the burst tails $(\sim 10 \%)$ is governed by processes other than magnetic burial, e.g. small-scale magnetic fields generated by an MHD dynamo in the burning front, which are confined to the ashes after the burst, while the freshly accreted matter remains unmagnetized (Spitkovsky et al. 2002).

Ageostrophic shear flow moves hot material ahead of the burning front and draws fresh fuel into it at the flame speed $v_{\text {flame }} \approx$ $2 \times 10^{7} \mathrm{~cm} \mathrm{~s}^{-1}\left(h_{\text {hot }} / 10^{3} \mathrm{~cm}\right)(f / 0.32 \mathrm{kHz})^{-1}\left(t_{\text {nuc }} / 0.1 \mathrm{~s}\right)^{-1}$, where $h_{\text {hot }}$ is the scale height of the incinerated ocean, $f=2 \Omega \cos \theta$ is the local Coriolis parameter, $\Omega$ is the angular frequency of the star, and $t_{\text {nuc }}$ is the nuclear burning time-scale. Magnetic tension stabilizes ageostrophic shear for $B \gtrsim\left(8 \pi \rho v_{\text {flame }}^{2}\right)^{1 / 2} \approx 4.5 \times 10^{10}$ $\mathrm{G} \ll B_{\max }$. In other words, when the cyclonic flame runs into the equatorial magnetic belt, it is reflected; conversely, ageostrophic shear cannot disrupt the belt. Latitudininal shear instabilities, a possible source of burst oscillations (Cumming 2005), may disrupt the magnetic belt and provide one motivation for extending our burial model to three dimensions in the future.

\section{DISCUSSION}

Polar magnetic burial creates an intense, equatorial belt of magnetic field which can thermally isolate the magnetic hemispheres of an accreting neutron star. The maximum magnetic field strength in the belt, $B_{\max }=$ $5.6 \times 10^{15}\left(M_{\mathrm{a}} / 10^{-4} M_{\odot}\right)^{0.32} \mathrm{G}$, is sufficient to prevent heat transport by conduction, radiation, convection, and ageostrophic shear. The conduction time-scale $t_{\text {cond }} \sim$ 27 yr exceeds the cooling time of the incinerated material; the magnetic belt is opaque (optical depth $\sim 7 \times 10^{4}$ ); convection is stabilized magnetically $\left(\rho v_{\mathrm{c}}^{2} \ll B_{\max }^{2} / 8 \pi\right)$; and so is ageostrophic shear $\left(\rho v_{\text {flame }}^{2} \ll B_{\max }^{2} / 8 \pi\right)$. However, the conclusion that the hemispheres are thermally isolated by the magnetic belt is less secure at large accreted masses $\left(M_{\mathrm{a}} \sim 0.1 M_{\odot}\right)$, where the Grad-Shafranov and ZEUS-3D calculations in section 2 are hampered by numerical difficulties, and Ohmic diffusion (which we do not incorporate self-consistently) becomes important.

Thermal isolation of the magnetic hemispheres is consistent with the highly sinusoidal light curves of millisecond oscillations in thermonuclear bursts in LMXBs (Muno et al. 2002). During the rise of the burst, oscillations are caused by the spreading of a hot spot, probably in the form of a cyclone (Spitkovsky et al. 2002). In the tail of the burst, the hot spot is sequestered in one hemisphere (Muno et al. 2002), and misalignment of the magnetic and spin axes guarantees that we observe persistent oscillations at the spin frequency. Furthermore, it is observed that bursts occasionally occur in quick succession, separated by a 5-10 minute interval, compared to an interval of several hours between typical bursts (Lewin et al. 1993). Such burst pairs are consistent with the model of magnetic burial: if a burst ignites one hemisphere, the other remains dormant and can, in principle, ignite shortly thereafter when more material accretes. If this explanation is valid, one would expect that there are no triple bursts, that the fluences emitted in each half of a burst pair are roughly equal, and that the number of burst pairs relative to normal bursts increases as the ignition probability per unit time increases (relative to $\left.\dot{M}_{\mathrm{a}}\right){ }^{1}$ A more detailed calculation is required to resolve whether the 10-min intervals are caused by delayed heat propagation through the magnetic belt.

Muno et al. (2002) showed that the bright burning region must cover $80^{\circ}-110^{\circ}$ in latitude in order to match the upper limit on the observed ratio of harmonic to fundamental amplitudes, which implies $\Delta \theta \lesssim 10^{\circ}$. If the scaling of $\Delta \theta$ versus $M_{\mathrm{a}}$ in $\$ 2.2$ is taken at face value, this requires $M_{\mathrm{a}}>0.5 M_{\mathrm{c}}$. Furthermore, for the belt to be optically thick, we need $M_{\mathrm{a}} \lesssim 2 M_{\mathrm{c}}$ (3.2). Apparently then, only a narrow range of accreted masses $\left(0.5 \lesssim M_{\mathrm{a}} / M_{\mathrm{c}} \lesssim 2\right)$ can account for the observations. However, the above $\Delta \theta$ scaling, which depends steeply on $M_{\mathrm{a}}$, does not tell the whole story, because Ohmic diffusion is not incorporated self-consistently. Equatorward hydromagnetic spreading is arrested when the accretion time-scale exceeds the Ohmic diffusion time-scale (Brown \& Bildsten 1998), softening the dependence of $B_{\max }$ and $\Delta \theta$ on $M_{\mathrm{a}}$ in the manner described by Melatos \& Pavne (2005). Ohmic diffusion arrests magnetic compression for $M_{\mathrm{a}}>M_{\mathrm{d}}$, where $M_{\mathrm{d}}=3.4 \times 10^{-7}\left(T / 10^{8} \mathrm{~K}\right)^{-2.2}\left(\dot{M}_{\mathrm{a}} / 10^{-8} M_{\odot} \mathrm{yr}^{-1}\right)^{0.44} M_{\odot}$ (Melatos \& Pavne 2005) is the accreted mass at which the accretion time-scale exceeds the Ohmic diffusion time-scale. Including this effect, $\Delta \theta$ is modified to the maximum of $3^{\circ}\left(M_{\mathrm{a}} / 10^{-4} M_{\odot}\right)^{-1.5}$ and $\left.3^{\circ}\left(M_{\mathrm{a}} / 10^{-4} M_{\odot}\right)^{0.5}\left(\dot{M}_{\mathrm{a}} / 10^{-8} M_{\odot} \mathrm{yr}^{-1}\right)^{-0.5}\right]$, and the belt remains optically thick even for $M_{\mathrm{a}} \gtrsim 2 M_{\mathrm{c}}$ (3.2).

Only 70 out of 160 LMXBs undergo bursts, and only 13 exhibit millisecond oscillations. Does the equatorial belt model respect these statistics? If accretion occurs at the Eddington rate, $\dot{M}_{\mathrm{a}} \approx 10^{-8} M_{\odot} \mathrm{yr}^{-1}$, it takes less than $10^{4}$ yr to achieve $M_{\mathrm{a}}>M_{\mathrm{c}}$ and screen the polar magnetic field, allowing bursts to ignite and creating a thermally insulating equatorial belt. However, for $M_{\mathrm{a}} \gtrsim 2 \times 10^{3} M_{\mathrm{c}}$, the belt becomes optically thin to Xrays and $t_{\text {cond }}$ drops below the duration of the burst, potentially allowing the flame to engulf the entire star in LMXBs older than a few times $10^{7} \mathrm{yr}$. If accretion occurs at $\dot{M}_{\mathrm{a}} \sim 10^{-11} M_{\odot \mathrm{yr}}{ }^{-1}$, as in accreting millisecond pulsars (Chakrabarty et al. 2003), it takes $10^{7} \mathrm{yr}$ to achieve $M_{\mathrm{a}}>M_{\mathrm{c}}$, screen the polar magnetic field, and allow bursts to ignite. Therefore, we do not expect bursts from all accreting millisecond pulsars, but when bursts do occur, we expect to detect oscillations at some level because we have $\tau_{\mathrm{R}} \gg 1$ and $t_{\text {cond }} \gg$ (burst time-scale) for $M_{\mathrm{a}} \lesssim 10^{-3} M_{\odot}$. Such is the case, within current observational sensitivity, for SAX J1808.4-3658

1 If the ignition probability is higher (given a specific $\dot{M}_{\mathrm{a}}$ ), it is more likely that the second hemisphere will ignite shortly after the first (before the first has a chance to refill and ignite again). A faster burst rate may be associated with more pairs, but gaps in the satellite data make it difficult to be sure. 
and XTE J1814-338. Sources like XTE J1814-338, whose oscillations contain harmonics exceeding 25 per cent of the peak amplitude (Strohmaver et al. 2003), may have semi-transparent equatorial belts; perhaps this millisecond pulsar has experienced interruptions in its accretion history resulting in $M_{\mathrm{a}}<M_{\mathrm{c}}$. Note that, for $\dot{M}_{\mathrm{a}} \lesssim 10^{-10} M_{\odot} \mathrm{yr}^{-1}$, Ohmic diffusion may magnetize the freshly accreted material (Cumming et al. 2001), increasing the polar magnetic field strength and suppressing bursts. Also, ignition is more likely near the equator, where gravity is rotationally reduced, but this is where the magnetic field is strongest. The issues of conservative mass-transfer (Tauris et al. 2000) and differential rotation leading to shear instabilities (Cumming 2005) are not considered here.

On the face of it, high-mass X-ray binaries (HMXBs) accrete many times $M_{\mathrm{c}}$, yet a strong magnetic field survives. However, one needs to be cautious. While the accretion rate in HMXBs can reach $10^{-10}-10^{-8} M_{\odot} \mathrm{yr}^{-1}$ during either atmospheric Roche lobe overflow (close binaries with orbital period $<5$ days) or stellar wind accretion (wider binaries), the typical lifetime of HMXBs as strong X-ray sources is $10^{4}-10^{5}$ yr (Urpin et al. 1998). Sub-Eddington accretion rates $\left(10^{-13}-10^{-10} M_{\odot} \mathrm{yr}^{-1}\right)$ characterize the rest of the companion's main-sequence evolution for $10^{6}-10^{7} \mathrm{yr}$. These scenarios yield roughly $M_{\mathrm{a}} \sim M_{\mathrm{c}}$. Mass transfer can also be nonconservative, further reducing $M_{a}$, e.g.. in intermediate-mass X-ray binaries (Tauris et al. 2000). Of course, despite these cautionary remarks, it may well be that some HMXBs do accrete many times $M_{\mathrm{c}}$. If so, then either HMXBs are a counterexample to the simple magnetic burial model we have calculated, or else cross-field transport by Ohmic diffusion (which we do not incorporate self-consistently) becomes important.
A recent model for doubly peaked bursts which are too weak to cause photospheric expansion (Bhattacharvva \& Strohmaver 2006) proposes that a burning front forms quickly after ignition at or near a pole and propagates quickly towards the equator where it stalls. After a delay, the burning front speeds up again into the opposite hemisphere. While the time between burst peaks ( $\sim 10$ seconds) is less than that estimated for burst propagation across the magnetic belt, a weak magnetic belt may explain why the burning front stalls at the equator.

Numerical difficulties associated with steep field gradients prevent us from verifying whether our model scales up to $M_{\mathrm{a}} \sim 0.1 M_{\odot}$. However, if we continue to respect mass-flux conservation as in 2.1 it is probable that accreting extra mass does not eliminate the magnetic barrier. Even if, for example, there are instabilities which disrupt the equatorial magnetic belt above $\sim 10^{-4} M_{\odot}$, the belt does not stay disrupted; magnetic burial ensures that it reforms as soon as a further $\sim 10^{-5} M_{\odot}$ is accreted (Pavne \& Melatos 2004). Given that $0.1 M_{\odot}$ is typically accreted in LMXBs, the chances of catching the belt in its disrupted state are slim.

We thank Duncan Galloway for pointing out to us that sinusoidal light curves are a signature of hemispheric emission, and alerting us to the existence of burst pairs. We thank an anonymous referee for pointing out to us that the burial model may imply the existence of pairs of bursts in quick succession, and for comments which improved the treatment of the crust and burning physics in the manuscript. This research was supported in part by an Australian Postgraduate Award.

\section{REFERENCES}

Bhattacharyya, S., \& Strohmayer, T. E. 2006, ApJ, 636, L121 Bhattacharyya, S., \& Strohmayer, T. E. 2006, ApJ, 641, L53

Bildsten, L. 1995, ApJ, 438, 852

Brown, E. F. \& Bildsten, L. 1998, ApJ, 496, 915

Brown, E. F. 2004, ApJ, 614, L57

Chakrabarty, D., Morgan, E. H., Muno, M. P., Galloway, D. K., Wijnands, R., van der Klis, M., \& Markwardt, C. B. 2003, Nat, 424,42

Cumming, A., Zweibel, E., \& Bildsten, L. 2001, ApJ, 557, 958

Cumming, A. 2005, ApJ, 630, 441

Fryxell, B. A. \& Woosley, S. E. 1982, ApJ, 258, 733

Galloway, D. K., Chakrabarty, D., Cumming, A., Kuulkers, E.,

Bildsten, L., \& Rothschild, R. 2004, AIP Conf. Proc. 714: X-ray

Timing 2003: Rossi and Beyond, 714, 266

Gough, D. O., \& Tayler, R. J. 1966, MNRAS, 133, 85

Heyl, J. S. 2004, ApJ, 600, 939

Joss, P. C. \& Li, F. K. 1980, ApJ, 238, 287

Lewin, W. H. G., van Paradijs, J., \& Taam, R. E. 1993, Space Science Reviews, 62, 223

Melatos, A. \& Phinney, E. S. 2001, PASA, 18, 421

Melatos, A. \& Payne, D. J. B. 2005, ApJ, 623, 1044

Miller, M. C. 1995, ApJ, 448, L29

Muno, M. P., Özel, F., \& Chakrabarty, D. 2002, ApJ, 581, 550

Özel, F. 2003, ApJ, 583, 402

Payne, D. J. B. \& Melatos, A. 2004, MNRAS, 351, 569
-. 2006, MNRAS, submitted

Piro, A. L. \& Bildsten, L. 2004, AAS/High Energy Astrophysics Division, 8

Potekhin, A. Y. 1999, A\&A, 351, 787

Schwarzschild, M. \& Härm, R. 1965, ApJ, 142, 855

Spitkovsky, A., Levin, Y., \& Ushomirsky, G. 2002, ApJ, 566, 1018

Stone, J. M. \& Norman, M. L. 1992, Astrophys. J., Suppl. Ser., 80, 753

Strohmayer, T. E. \& Bildsten, L. 2003, To appear in Compact Stellar X-Ray Sources, eds. W.H.G. Lewin and M. van der Klis, Cambridge University Press

Strohmayer, T. E., Jahoda, K., Giles, A. B., \& Lee, U. 1997, ApJ, 486,355

Strohmayer, T. E., Markwardt, C. B., Swank, J. H., \& in't Zand, J. 2003, ApJ, 596, L67

Tauris, T. M., van den Heuvel, E. P. J., \& Savonije, G. J. 2000, ApJ, 530, L93

Urpin, V., Konenkov, D., \& Geppert, U. 1998, MNRAS, 299, 73

Wijnands, R., van der Klis, M., Homan, J., Chakrabarty, D., Markwardt, C. B., \& Morgan, E. H. 2003, Nat, 424, 44

Zingale, M., Timmes, F. X., Fryxell, B., Lamb, D. Q., Olson, K., Calder, A. C., Dursi, L. J., Ricker, P., Rosner, R., MacNeice, P., \& Tufo, H. M. 2001, Astrophys. J., Suppl. Ser., 133, 195 

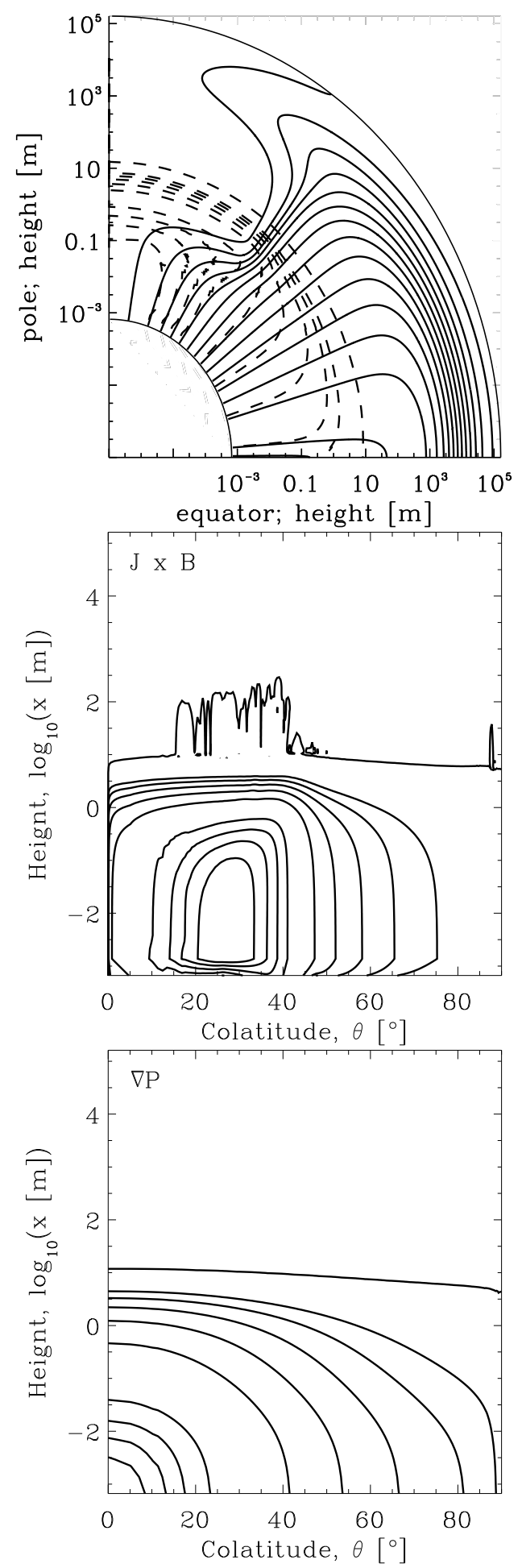

FIG. 1.- (top) Equilibrium magnetic field lines (solid curves) and density contours (dashed curves) for $M_{\mathrm{a}}=10^{-5} M_{\odot}$ and $\psi_{\mathrm{a}}=0.1 \psi_{*}$. Coordinates measure altitude. Density contours are drawn for $\eta \rho_{\max }\left(\rho_{\max }=2.52 \times 10^{14} \mathrm{~g} \mathrm{~cm}{ }^{-3}\right)$, with $\eta=$ 0.8, 0.6, 0.4 $0.2,10^{-2}, 10^{-3}, 10^{-4}, 10^{-5}, 10^{-6}, 10^{-12}$. Convergence residuals are less than $10^{-3}$. [From Pavne \& Melatos (2004).] (middle) Contours of Lorentz force per unit volume for the same $\eta$ values. (bottom) Contours of pressure gradients for the same $\eta$ values. Note that colatitude $=0^{\circ}$ at the pole. 


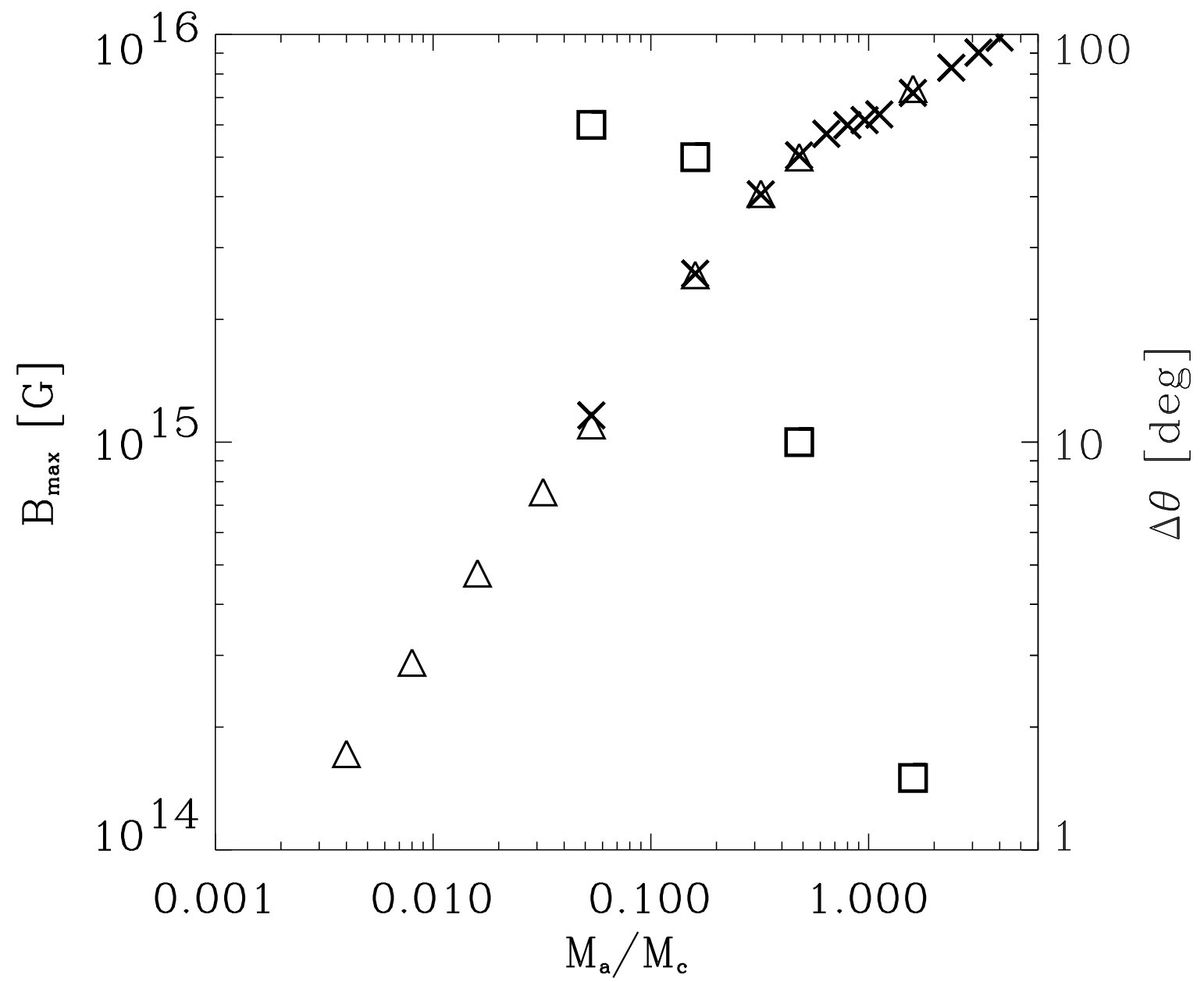

FIG. 2.- Maximum magnetic field in the equatorial belt, $B_{\max }$, computed numerically for $h / R_{*}=2 \times 10^{-2}($ crosses $)$ and $h / R_{*}=5 \times 10^{-5}$ (triangles) and scaled using $B_{\max } \propto h^{-1}$, plotted together with the half-width half-maximum thickness, $\Delta \theta$ (squares), as a function of accreted mass, $M_{\mathrm{a}}$. 
8

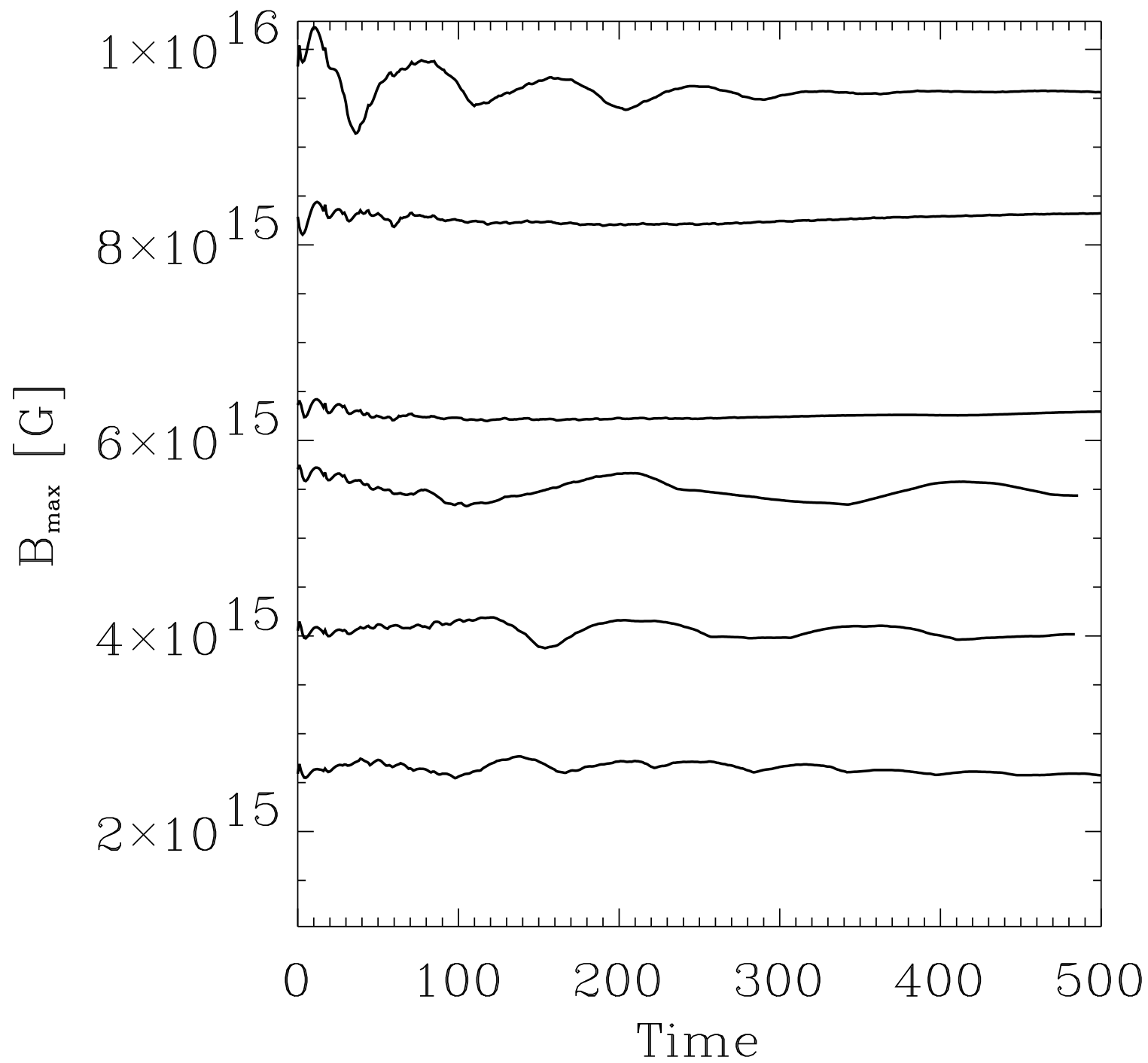

Fig. 3.- Maximum magnetic field strength, $B_{\max }$, as a function of time (in units of the Alfvén time) for $M_{\mathrm{a}} / M_{\mathrm{c}}=$ $0.16,0.32,0.64,1.12,2.4,4.0$ (bottom to top) when the equilibrium in Figure 1 is loaded into ZEUS-3D and perturbed slightly. The configuration is stable. 